\title{
BMJ Open Cardiovascular, antidepressant and immunosuppressive drug use in relation to risk of cutaneous melanoma: a protocol for a prospective case- control study
}

\author{
Leon Alexander Mclaren Berge, ${ }^{1}$ Bettina Kulle Andreassen, ${ }^{1}$ \\ Jo Steinson Stenehjem, ${ }^{\oplus 1,2}$ Inger Kristin Larsen, ${ }^{1}$ Kari Furu, ${ }^{3}$ Asta Juzeniene, ${ }^{4}$ \\ Ingrid Roscher, ${ }^{5}$ Trond Heir, ${ }^{6}$ Adele Green, ${ }^{7}$ Marit Bragelien Veierød, ${ }^{2}$ \\ Trude Eid Robsahm ${ }^{1}$
}

To cite: Berge LAM, Andreassen BK, Stenehjem JS, et al. Cardiovascular, antidepressant and immunosuppressive drug use in relation to risk of cutaneous melanoma: a protocol for a prospective case-control study. BMJ Open 2019;9:e025246. doi:10.1136/ bmjopen-2018-025246

- Prepublication history and additional material for this paper are available online. To view these files, please visit the journal online (http://dx.doi org/10.1136/bmjopen-2018025246).

Received 5 July 2018 Revised 4 January 2019 Accepted 11 January 2019

Check for updates

(C) Author(s) (or their employer(s)) 2019. Re-use permitted under CC BY-NC. No commercial re-use. See rights and permissions. Published by BMJ.

For numbered affiliations see end of article.

Correspondence to

Leon Alexander Mclaren Berge; Leon.Berge@kreftregisteret.no

\section{ABSTRACT}

Introduction The incidence of cutaneous melanoma (hereafter melanoma) has increased dramatically among fair-skinned populations worldwide. In Norway, melanoma is the most rapidly growing type of cancer, with a $47 \%$ increase among women and $57 \%$ among men in 2000-2016. Intermittent ultraviolet exposure early in life and phenotypic characteristics like a fair complexion, freckles and nevi are established risk factors, yet the aetiology of melanoma is multifactorial. Certain prescription drugs may have carcinogenic side effects on the risk of melanoma. Some cardiovascular, antidepressant and immunosuppressive drugs can influence certain biological processes that modulate photosensitivity and immunoregulation. We aim to study whether these drugs are related to melanoma risk.

Methods and analysis A population-based matched casecontrol study will be conducted using nation-wide registry data. Cases will consist of all first primary, histologically verified melanoma cases diagnosed between 2007 and 2015 identified in the Cancer Registry of Norway (14 000 cases). Ten melanoma-free controls per case (on date of case melanoma diagnosis) will be matched based on sex and year of birth from the National Registry of Norway. For the period 2004-2015, and by using the unique personal identification numbers assigned to all Norwegian citizens, the case-control data set will be linked to the Norwegian Prescription Database for information on drugs dispensed prior to the melanoma diagnosis, and to the Medical Birth Registry of Norway for data regarding the number of child births. Conditional logistic regression will be used to estimate associations between drug use and melanoma risk, taking potential confounding factors into account. Ethics and dissemination The project is approved by the Regional Committee for Medical Research Ethics in Norway and by the Norwegian Data Protection Authority. The study is funded by the Southeastern Norway Regional Health Authority. Results will be published in peer-reviewed journals and disseminated further through scientific conferences, news media and relevant patient interest groups.

\section{Strengths and limitations of this study}

- Linkage between four nation-wide population-based registries through unique personal identification numbers produces comprehensive, complete and high-quality data for analysis.

- A high number of melanoma cases with information on drug use prior to the melanoma diagnosis further enhances the strength of the study.

The latency time between drug exposure and melanoma diagnosis is uncertain and in the case of this study, it may not be sufficient to infer a relation between drug use and cancer development.

- Data pertaining to measures of residential ambient ultraviolet exposure is available, but data on recreational sun exposure, everyday sun exposure, sunburn, solarium, family history of melanoma, educational level, anthropometry and hormone use as potential confounders are lacking.

\section{INTRODUCTION}

Rationale and evidence gaps

Cutaneous melanoma (hereafter melanoma) is the most lethal form of skin cancer. During the period 2000-2016, a remarkable increase in the age-standardised incidence of melanoma has been seen in Norway, with a $57 \%$ and $47 \%$ increase among men and women, respectively, making melanoma the fastest growing malignancy in Norway. ${ }^{1}$ Norway is ranked among the top five worldwide in age-standardised melanoma incidence rates, years of healthy life lost and mortality. ${ }^{2}$

Ultraviolet (UV) radiation from sun and solarium, which is classified as a human carcinogen by the International Agency for Research on Cancer (IARC), ${ }^{34}$ was responsible for approximately $75.7 \%$ of all new 
melanoma cases worldwide in 2012. ${ }^{5}$ The development of melanoma is, however, a multifactorial process, with risk also depending on individual susceptibility. These include certain phenotypic characteristics, ${ }^{6}$ a previous melanoma diagnosis, ${ }^{7}$ family history of melanoma, ${ }^{8}$ anthropometry, ${ }^{9}$ hormone factors ${ }^{10}$ and likely alcohol consumption. $^{11}$

Other factors may also influence melanoma development and contribute to its steady increase. Results from etiological studies indicate that exposure to and use of commonly prescribed drugs may represent such a factor (see online supplementary tables S1-S3). Drug safety has high priority and the European Medicines Agency has recently improved their systems, Exploring and Understanding Adverse Drug Reactions (EU-ADR) in the European Union, for active surveillance of adverse drug events. However, the EU-ADR is not ideal for capturing adverse events with long latency, such as cancer, because long-term monitoring is not part of the drug programme. Similar limitations apply for the US Food and Drug Administration (FDA). Consequently, knowledge on the possible carcinogenicity of marketed drugs is sporadic or lacking.

Pharmacoepidemiological studies and meta-analyses have contributed to establishing evidence of the carcinogenicity of drugs. Since 1970, IARC has performed comprehensive and systematic reviews of animal, laboratory, mechanistic and epidemiological studies to evaluate the carcinogenicity of drugs. Group 1 agents are those considered carcinogenic to humans, whereas groups $2 \mathrm{a}$ and $2 \mathrm{~b}$ are agents with probable and possible carcinogenic effects, respectively. ${ }^{12}$ However, many commonly used drugs have not been evaluated due to lack of longterm monitoring.

Some drugs can have skin carcinogenic potential, directly through a biological mechanism of the drug itself, which may include functional alterations of the immune system and the tumour microenvironment, and/ or through an interaction with UV exposure, resulting in increased photosensitivity. ${ }^{13}$ Drugs that could play a role in melanoma development through such mechanisms include some cardiovascular, antidepressants and immunosuppressive drugs although present studies do not show unanimous results (see online supplementary tables S1-S3). From 2005 to 2015, the number of people in Norway prescribed cardiovascular drugs rose from over 800000 to over 1000000 (excluding inpatient use). The same numbers were 275000 to about 330000 for antidepressants and 26000 to 55000 for immunosuppressive drugs. ${ }^{15} 16$

The results of most studies warrant the need for further analyses with more detailed information on drug use and confounders to elucidate relations between these drug types and cancer. ${ }^{17}$ Whether or not any drugs of these types have an association with the incidence of melanoma is highly important as the number of people receiving these drugs is increasing.

\section{Cardiovascular drugs}

Several types of cardiovascular drugs, including $\beta$-blocking agents, diuretics, ACE inhibitors (ACEi) and angiotensin receptor blockers (ARBs), may influence melanoma development (see online supplementary table S1). A biological basis for the role of $\beta$-blockers in melanoma progression exists, as melanoma tissue expresses both $\beta 1$ - and $\beta 2$-adrenoreceptors. These, in turn, are known to stimulate the production of vascular endothelial growth factor, interleukin- 6 and interleukin- 8 , which promote angiogenesis and tumour growth. ${ }^{18}$ Long-term exposure to $\beta$-blockers has been associated with a reduced risk of melanoma progression, ${ }^{19}$ melanoma recurrence and death. ${ }^{20}{ }^{21}$ On the other hand, a meta-analysis of studies found that $\beta$-blockers and diuretics might be positively associated with melanoma, ${ }^{22}$ which has been supported by a recent meta-analysis of cohort studies, case-control studies, and randomised clinical trials. ${ }^{17}$

Diuretics have been shown to have photosensitising potential $^{23}$ and use of the diuretics indapamide and thiazide has been found to increase the risk of melanoma ${ }^{22}$ 24-26 though no such association was found in a recent meta-analysis. ${ }^{17}$ Another recent analysis regarding the use of the diuretic hydrochlorothiazide found no association with melanoma in general, stratification by histological subtype however, revealed positive associations with the subtypes nodular and lentigo melanoma. ${ }^{27}$ Use of statins however, another prominent drug group, has been associated with decreased melanoma progression. ${ }^{28}$

ACE may also be involved in cancer processes through regulation of cell proliferation and migration. ${ }^{29}$ It remains unclear, whether ACEi or ARBs influence melanoma development. A review of observational and interventional studies indicated that ACEi and ARBs positively affect survival in melanoma patients. ${ }^{30}$ A recent meta-analysis, however, found that neither ACEi nor ARBs were associated with any form of skin cancer. ${ }^{17}$

\section{Antidepressant drugs}

In a comprehensive European case-control study of known and potentially new risk factors for skin cancer, stress, traumatic events and depression were identified as significant risk factors for melanoma. ${ }^{31}$ This relation can result from the biological effects of stress but also raises the question of whether it is the result of other factors like associated drug use.

Laboratory and animal studies have found cancer-promoting effects of antidepressants ${ }^{32}$ while for melanoma, in particular, few studies exists (see online supplementary table S2). Major types of antidepressants include selective serotonin reuptake inhibitors (SSRI), non-selective monoamine reuptake inhibitors (NSMRI), monoamine oxidase inhibitors and tricyclic antidepressants (TCA). The SSRI sertraline displays cytotoxicity against human melanoma cell lines through downregulating the pro-survival molecule Akt that normally prevents cell death through apoptosis. ${ }^{33}$ High-dose sertraline (75-fold to 100 -fold higher than clinical doses) also has the capacity 
to reduce protein synthesis and thus cell proliferation, giving it antineoplastic properties. ${ }^{34}$

Fluoxetine, another SSRI, has been found to induce melanogenesis in melanoma cell lines in vitro and in vivo, ${ }^{35}$ and it is associated with an increased number of brain metastases from breast cancer in mice. ${ }^{36}$ On the other hand, animal studies have demonstrated that fluoxetine significantly inhibits melanoma tumour growth and melanoma-induced oxidative changes through antioxidant activity. ${ }^{37} 38$ The TCAs amitriptyline, nortriptyline and clomipramine have previously displayed an ability to inhibit the growth of melanoma cell lines and primary cell cultures in vitro. ${ }^{39}$ The TCA desipramine is also demonstrated to inhibit melanoma tumour growth in vivo. ${ }^{40}$

\section{Immunosuppressive drugs}

Immunosuppressive drugs are used to prevent rejection following organ transplantation and for treatment of autoimmune disorders. These drugs have several well-documented side effects, of which infections and cancer are the most frequent due to the nonspecific nature of the immune suppression. ${ }^{41}$ A well-known side effect is significantly increased risk of non-melanoma skin cancer, ${ }^{42}$ but a positive association with melanoma risk and mortality have also been observed (see online supplementary table S3). ${ }^{43}$

A systematic review of the FDA adverse events reporting system and of medical records detected a significant association between tumour necrosis factor- $\alpha$ inhibitors and increased melanoma risk. The drugs identified as having an association with melanoma were the monoclonal antibodies, such as infliximab, adalimumab and golimumab, as well as the receptor fusion protein etanercept. ${ }^{44}$ Glucocorticoids, another group of immunosuppressive agents, have been found to inhibit melanoma growth. ${ }^{45} 46$

The antiproliferative agent azathioprine causes accumulation of 6-thioguanine in DNA. These components are thought to work synergistically with UVA radiation to generate reactive oxygen species with mutagenic potential. ${ }^{47}$ This propensity to increase UV-induced DNA damage is suggested to be responsible for the development of melanoma in users of azathioprine. ${ }^{48}$

A large and comprehensive population-based study using nation-wide registry data provides a unique opportunity to explore the impact of the drug types in question on melanoma risk. To our knowledge, a similar study has not been conducted, making the current research question a significant matter for public health systems worldwide.

\section{Aims and hypothesis}

The central hypothesis of this project is that use of cardiovascular, antidepressant and immunosuppressive drugs increases the risk of melanoma. With this study protocol, we propose a population-based case-control study with the aim of examining this hypothesis with the following questions:

1. Is use of prescribed cardiovascular drugs (in particular diuretics) associated with melanoma risk?

2. Is use of prescribed antidepressants associated with melanoma risk?

3. Is use of prescribed immunosuppressive drugs and/or monoclonal antibodies associated with melanoma risk?

\section{Methods and analysis}

This project will be carried out by merging data from four Norwegian national population-based registries (figure 1) with complete and high-quality data due to mandatory reporting by law. The unique personal identification number (PIN) issued to all Norwegian residents on birth or immigration enables data linkage across the registries. The study sample will encompass approximately 14 000 melanoma cases with 10 matched controls per case, alongside data regarding pre-diagnostically dispensed cardiovascular antidepressant and immunosuppressive

\section{Source population}

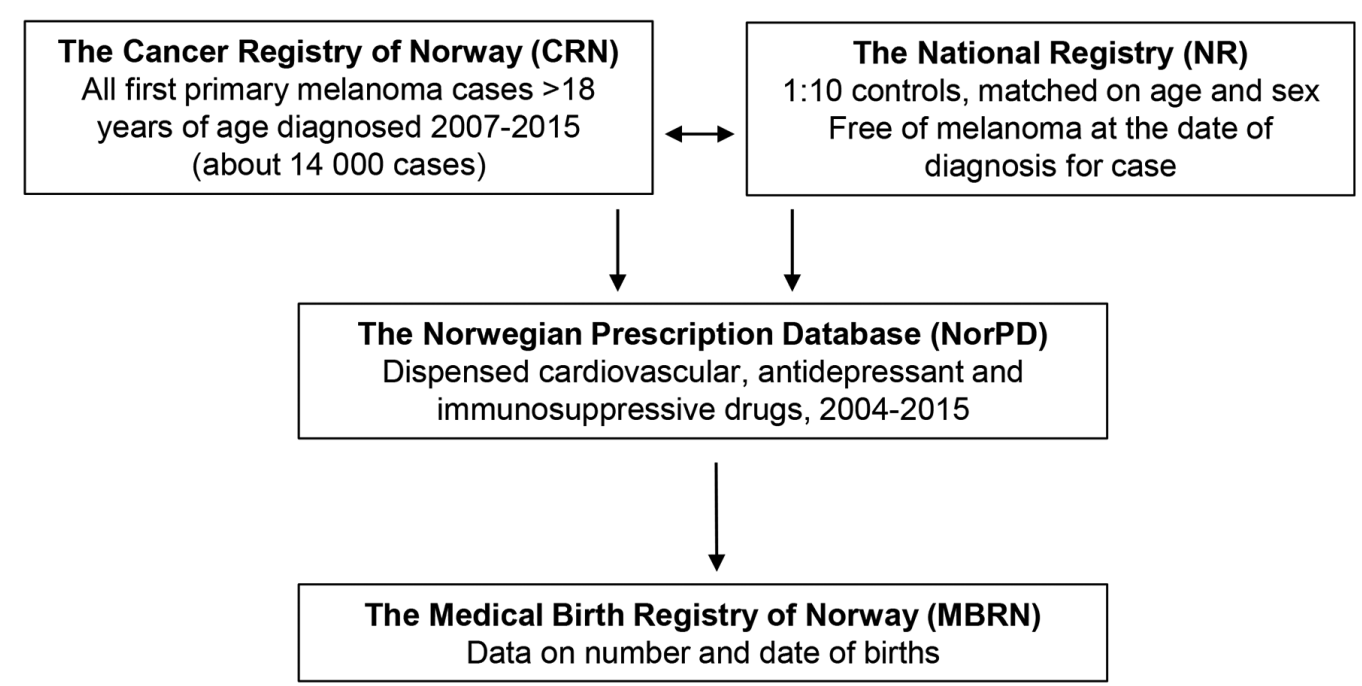

Figure 1 A diagram illustrating the source population and the data to be obtained from each of the four nation-wide registries. 
drugs, including data regarding number and dates of child births.

\section{Patient and public involvement}

As the study proposed by the protocol in question is register based, the research question and outcome measures were not informed by any specific patient priorities, experiences or preferences. Rather, their formulation was based on our own priorities for patient benefit and result interpretation. The case-control study described by the protocol uses only data from nation-wide population-based registers and thus will not include a recruitment process for patients, who will not be involved in neither the design nor conduct of the study. All results will be distributed via the news media, relevant patient and drug user groups, as well as peer-reviewed journals and scientific conferences. The study described by the protocol in question is not a randomised control trial and will not have measures of intervention that could burden patients in any way assessable.

\section{The registries}

The Cancer Registry of Norway (CRN) has registered information on all cancers diagnosed in Norway since 1953. The registry receives data from several independent sources (medical practitioners, pathology laboratories and the Cause-of-Death Registry) ensuring complete and up-to-date high-quality data. ${ }^{49}$ Cancer diagnoses are recorded using the International Classification of Disease version 10. For our analyses, we will obtain the following data on all first-time melanoma cases, diagnosed in the age group 18-85 years between 2007 and 2015: sex, age at diagnosis, date of diagnosis, tumour location, histopathological factors (histological type, anatomic location (see online supplementary table S4), Breslow thickness (since 2008), clinical stage and ulceration) and place of residence. Case-by-case data regarding Breslow thickness is missing from all diagnoses in 2007 but will be included through imputation in order to study Breslow thickness as an outcome.

The National Registry contains information on births, citizenship, change of address and migration to and from Norway with dates, for all citizens, which allows for the sampling of general population controls and tracking of all study subjects. The Norwegian Prescription Database (NorPD) contains information on all prescribed medications (reimbursed or not), dispensed at pharmacies to individual patients treated in ambulatory care from 1 January 2004 in the entire Norwegian population (5.3 million individuals in 2018). In NorPD, the information available for each dispensed drug is the Anatomical Therapeutic Chemical (ATC) classification code, substance name, trade name, pharmaceutical formulation, strength, package size, number of packages, amount dispensed in Defined Daily Doses, reimbursement code and dispensing date. ${ }^{50}$

Drugs supplied in hospitals and nursing homes are not included at the individual level in NorPD. All drugs dispensed are classified according to the WHO ATC classification. ${ }^{51}$ For the purpose of our analyses, we will obtain information on use of cardiovascular (and in particular diuretic) drugs (ATC code: C01-C10), antidepressant drugs (ATC code: N06A), immunosuppressive (ATC code: L04) drugs (see online supplementary table S4), as well as the use of other drug types. All drugs in question are prohibited for sale in Norway without an associated prescription from a physician. The drugs of each type considered for the analysis will be limited to those where the amount of available patient user data can facilitate statistically significant data analysis. Data from region-specific UV measurement stations will be obtained from the Norwegian Radiation Protection Authority to calculate ambient lifetime cumulative UV dose according to county of residence at the time of diagnosis. ${ }^{52}$ The Medical Birth Registry of Norway (MBRN) was established in 1967 and has since recorded information on all deliveries in Norway. Data to be obtained for all cases and controls are number and dates for births experienced until the point of diagnosis (cases) or index date (controls).

\section{Study design}

Using a nested case-control design, we will explore the melanoma incidence and level of multiple drug exposures in melanoma cases and controls. Furthermore, we will investigate whether drug use is related to melanoma risk, as well as to histological subtype, clinical stage, Breslow thickness, ulceration and ambient UV exposure of residence through stratified analyses. Cases will consist of all first primary histologically verified melanomas (18-85 years) diagnosed in Norway in the period 2007-2015 (figure 2). In all, 10 controls per case (1:10) will randomly be selected from the general population, alive and free of cancer at the date of diagnosis (index

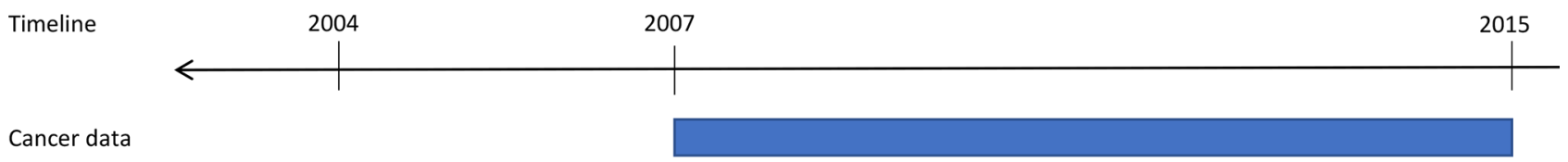

Drug dispensed

Birth data

Figure 2 A timeline illustrating from which time periods the relevant data are to be obtained for the study. 
Table 1 Overview of case, control and matching criteria for the study sample

\begin{tabular}{|c|c|}
\hline Case criteria & Study criteria \\
\hline Cases & $\sim 14000$ \\
\hline Verification & $\begin{array}{l}\text { Histological or cytological verified } \\
\text { melanoma (ICD-10: C43) }\end{array}$ \\
\hline Definition & $\begin{array}{l}\text { Norwegian inhabitants with a } \\
\text { diagnosis of invasive melanoma } \\
\text { without a history of cancer }\end{array}$ \\
\hline Age at diagnosis & 18-85years \\
\hline Year of diagnosis & 2007-2015 \\
\hline Sex & Male and female \\
\hline \multicolumn{2}{|l|}{ Control criteria } \\
\hline Controls & 140000 (1:10 matching) \\
\hline Definition & $\begin{array}{l}\text { Alive, resident in Norway with no } \\
\text { history of cancer before respective } \\
\text { case diagnosis }\end{array}$ \\
\hline Selection & $\begin{array}{l}\text { Random sampling within matching } \\
\text { criteria (with replacement) from a } \\
\text { pool of available population }\end{array}$ \\
\hline \multicolumn{2}{|l|}{ Matching criteria } \\
\hline Sex & Same sex as case \\
\hline Age at diagnosis & Same year of birth as case \\
\hline Index date & $\begin{array}{l}\text { Alive and free of cancer at the date of } \\
\text { diagnosis (case) }\end{array}$ \\
\hline
\end{tabular}

ICD-10, International Classification of Disease version 10.

date) for the case, and matched on sex and year of birth (risk set sampling). Table 1 gives the description of case, control and matching criteria.

Any case which is found to have two or more simultaneous diagnoses of melanoma will be removed from the main analysis in addition to their respective controls. This subgroup may, however, constitute an additional subject of investigation given that its numbers can facilitate a statistical analysis of sufficient power. Exposure to a particular drug or drug group among all cases and controls will be assessed from drugs dispensed as recorded in NorPD from 2004 to 2015 (figure 2). First, drug exposure will be defined as chronic drug use, that is, the dispensing of a drug which covers at least 2 years of use before the index date. Second, the cumulative dose will be assessed based on the number of prescriptions, total dose and duration of use, for each drug group. Third, drug exposure will be modelled as a time-dependent exposure by categorising the drug use at each time point as nonuser, user and past user. NorPD has registered dispensed prescription drugs from 1 January 2004. To account for the uncertainty of drug use before this date, we will apply a 6 -month quarantine from 1 January2004 to 30 June 2004. Thus, we will exclude all individuals with drug use within this time frame. Alternatively, we will use all registered dispensed drugs after 1 January 2004 and adjust for drug use within the time period from 1 January 2004 to 30 June 2004. Drug groups will be categorised into therapeutic subgroups (ATC second level). These subgroups will additionally be categorised by pharmacological subgroups (ATC fourth level) and chemical substances (ATC fifth level) to account for the potential confounding introduced by the different indications for which the drugs of interest can be given. ${ }^{53}$ Thus, where applicable with regard to statistical power, this will allow for the comparison of effects between subgroups and enable the use of active comparators as controls for specific agents of interest. To reduce confounding by indication, an additional covariate pertaining to the dispensation of other drug types prior to index date in addition to cardiovascular, antidepressant and immunosuppressive drugs will be implemented as a proxy for general healthcare usage among cases and controls.

Accounting for a certain latency period is prudent when assigning cancer development to some drug types as it reduces the possibility of reverse causation bias. On the other hand, certain drugs may have cancer-promoting properties which mediate late steps in the carcinogenesis. ${ }^{54}$ Other studies have also demonstrated the potential for relatively immediate effects of interventions designed to mediate the risk of melanoma. ${ }^{55}$ To account for this, the analyses will be conducted with and without consideration for a 1-, 3- and 5-year latency period between drug use and melanoma diagnosis. Additionally, as a lag period after drug discontinuation covers the latent period in which the effects of the drug in focus may still manifest, the time after drug discontinuation will also be considered time at risk with regard to attributing carcinogenic or anticarcinogenic properties to drugs.

\section{Statistical methods}

As the study will have a nested case-control design with risk set sampling (1:10 matching), conditional logistic regression analysis will be the main statistical method, estimating ORs and 95\% CIs for the association between melanoma and the drug in focus. Drug use will be modelled as a binary (chronic drug use) and continuous (cumulative dose) variable (see above).

In the analyses of drug use in relation to anatomic location of the tumour, we will test whether exposure-disease associations differ by sites by a contrast test. The same approach will be used in a stratified analysis of drug use and its associations with histopathological subtypes, clinical stage, Breslow thickness and ulceration (since 2008; in $\mathrm{T}$ categories ${ }^{56}$ ). We will also perform a linear regression analysis, using the Breslow thickness of melanoma as a continuous outcome variable among cases only. Due to the skewed distribution of Breslow thickness, $\log _{\mathrm{e}}$-transformation will be used and back-transformed estimates (geometric means) will be presented. ${ }^{9}$

We will adjust for residential ambient UV exposure according to lifetime cumulative UV dose. ${ }^{9}$ We will also categorise region of residence as urban or rural areas to indicate dermatologist availability. Number of births is also a potential covariate in the analyses. We will test for 
Table 2 The minimum OR detectable according to proportion of controls exposed to a particular drug type, using a power of $80 \%$ and a significance level of 0.05

\begin{tabular}{llllc}
\hline $\begin{array}{l}\text { Proportion } \\
\text { of exposed } \\
\text { controls (\%) }\end{array}$ & OR & $\begin{array}{l}\text { Number of } \\
\text { cases }\end{array}$ & $\begin{array}{l}\text { Number of } \\
\text { controls }\end{array}$ & $\begin{array}{l}\text { Total study } \\
\text { population }\end{array}$ \\
\hline 5 & 1.1 & 18902 & 189020 & 207922 \\
\hline 5 & 1.2 & 4904 & 49040 & 53944 \\
\hline 5 & 1.3 & 2257 & 22570 & 24827 \\
\hline 10 & 1.1 & 10041 & 100410 & 110451 \\
\hline 10 & 1.2 & 2622 & 26220 & 28842 \\
\hline 10 & 1.3 & 1214 & 12140 & 13354 \\
\hline 20 & 1.1 & 5722 & 57220 & 62942 \\
\hline 20 & 1.2 & 1513 & 15130 & 16643 \\
\hline 20 & 1.3 & 709 & 7090 & 7799 \\
\hline
\end{tabular}

relevant interactions such as sex/drugs, urban or rural residence/drugs as well as number of births/drugs. The significance level will be set to $5 \%$ and all statistical analyses will be performed using the $\mathrm{R}$ Statistical Software Package (V.3.5.1). ${ }^{57}$

\section{Power and sample size calculations}

The statistical power was set to $80 \%$ with a significance level of $5 \%$. Calculations were performed using R. Table 2 shows the minimum OR detectable for different sample sizes under the assumption that various proportions of controls are using a particular type of drug. Due to the size of the study samples for each study $(n=154000)$ including 14000 melanoma cases, we have enough statistical power to detect an OR of at least 1.2, assuming that $5 \%$ of the controls are exposed to the drug in question. Alternatively, an OR of 1.1 can also be achieved if at least $10 \%$ or $20 \%$ of controls have been exposed to the particular drug in question.

\section{Analysis plan}

In order to test the hypotheses above, the following analyses will be conducted:

1.1: A matched case-control analysis of overall melanoma risk according to the exposure and level of use of prescribed cardiovascular drugs (diuretics in particular). 1.2: A matched case-control analysis of melanoma risk stratified by anatomic site, histopathological subtype, clinical stage, Breslow thickness, ulceration and residential ambient UV exposure, according to the exposure and level of use of prescribed cardiovascular drugs (diuretics in particular).

2.1: A matched case-control analysis of melanoma risk according to the exposure and level of use of prescribed antidepressant drugs.

2.2: A matched case-control analysis of melanoma risk stratified by anatomic site, histopathological subtype, clinical stage, Breslow thickness, ulceration and residential ambient UV exposure, according to the exposure and level of use of prescribed antidepressant drugs.
3.1: A matched case-control analysis of melanoma risk according to the exposure and level of use of prescribed immunosuppressive drugs and/or monoclonal antibodies.

3.2: A matched case-control analysis of melanoma risk stratified by anatomic site, histopathological subtype, clinical stage, Breslow thickness, ulceration and residential ambient UV exposure, according to the exposure and level of use of prescribed immunosuppressive drugs and/ or monoclonal antibodies.

4: A linear regression analysis examining the Breslow thickness of melanoma as a continuous outcome, among cases only, according to the exposure and level of use of prescribed drugs.

\section{Project strengths and limitations}

Each analysis relies on high-quality data collected from nation-wide population-based health registries from 2004 to 2015 , with mandatory reporting and linkage secured by the PINs. This level of detail lends itself well to this prospective case-control study and allows us to take into account a wide range of variables for a high level of resolution in the statistical analyses. While recall bias represents a frequent limitation to the case-control design, all exposure data for the analysis will have been collected before the outcome. Hence, the use of prospectively collected high-quality data, without the need for personal recollection, eliminates the risk of recall bias.

While we will assume that drugs were used on the same date at which they were dispensed from the NorPD, it is not known, for certain, whether the drugs in question were used at this time. However, because only information pertaining to drug dispensation and purchase by patients is recorded in the NorPD, primary nonadherence is not an issue. ${ }^{58}$ The NorPD only records information on all prescribed drugs dispensed to individual patients from all pharmacies in Norway, excluding nonprescribed drugs and drugs dispensed to inpatients in hospitals or institutions. However, given the size and quality of our data from the general population, it is unlikely that this limitation will significantly influence the main results of our study. Additionally, as reporting to the respective registers is mandatory by law, the problem of selection bias is therefore negligible. Underlying indications for drug use might influence the risk of melanoma and may introduce potential confounding by indication. In addition to the use of cardiovascular, antidepressant and immunosuppressant drugs, we will account for the use of other drug types in our analyses, which will simultaneously act as a proxy indicator of potential differences in healthcare usage.

The main limitation is the potentially short latency time between drug use and melanoma diagnosis that this study allows for. The NorPD holds individual data on prescribed drugs dispensed to individuals since 1 January 2004, which can result in a short latency time for cancer development and detection throughout 20072015. The exposure window for most cancer-drug associations is unknown, though a quantitative analysis of the 
genetic evolution of pancreatic cancer found a 17-year gap between the initial carcinogenic mutation and the acquisition of metastatic capabilities by the primary tumour. ${ }^{59}$ The time between initial carcinogenesis and clinical detection of many cancers is also assumed to be long (10-30 years in some cases), and cancer is thus not an immediate effect of drug exposure ${ }^{13}$ The long period of cancer development, the latency of any carcinogenic and antineoplastic drug effects and unknown biological mechanisms of efficacy all contribute to the considerable time it takes to fully elucidate potential drug-cancer relationships. Additionally, while we will adjust for residential ambient UV exposure, we will not be able to account for other UV exposure variables such as recreational sun exposure, sunburns (as a marker of episodes of severe acute UV exposure) or indoor tanning. Neither will we be able to take phenotypic characteristics (fair complexion, freckles and nevi), socioeconomic variables (eg, education, occupation), healthcare utilisation, comorbidity, postmenopausal hormone use and anthropometric factors into account, which may represent confounding sources of individual-level exposure.

\section{ETHICS AND DISSEMINATION}

The linkage key for the 11-digit PINs will be stored and governed by a third party unavailable to the research team. All data management and analyses will be conducted on encrypted data with no individual persons identified.

This project can generate new and important knowledge on risk factors for melanoma and about melanoma aetiology, for better and more targeted prevention measures both in Norway and internationally. Our results can be of high importance for users of prescribed drugs and for the design of public health campaigns and future surveillance programmes, specifically addressing patients with a risk profile that predisposes for development of melanoma.

All results will be published in international peer-reviewed journals and presented at national and international conferences. The results will also be communicated directly to relevant user groups such as the Norwegian Cancer Society, The Norwegian Melanoma Association and other interest groups for patients that would be dependent on the drugs in question. Annual Norwegian conferences and seminars will serve as additional platforms for the distribution of knowledge to clinicians and researchers. Furthermore, a project-specific website, social media and other potential channels will also serve as platforms to distribute relevant results to patients and the general population.

\footnotetext{
Author affiliations

${ }^{1}$ Department of Research, Kreftregisteret, Oslo, Norway

${ }^{2}$ Department of Biostatistics, Universitetet i Oslo Institutt for medisinske basalfag, Oslo, Norway

${ }^{3}$ Department of Pharmacoepidemiology, Nasjonalt folkehelseinstitutt, 0slo, Norway ${ }^{4}$ Department of Radiation Biology, Oslo Universitetssykehus Institutt for kreftforskning, Oslo, Norway
}

${ }^{5}$ Department of Rheumatology, Dermatology and Infectious Diseases, Oslo University Hospital, Oslo, Norway

${ }^{6}$ Section for Trauma, Catastrophes and Forced Migration - Adults and Elderly, Nasjonalt kunnskapssenter om vold og traumatisk stress AS, Oslo, Norway ${ }^{7}$ QIMR Berghofer Medical Research Institute, Herston, Queensland, Australia

Contributors TER conceived the study. TER, BKA, MBV and AJ contributed to the project design. LAMB and TER drafted the manuscript while BKA, MBV, JSS, IKL, KF, $A J, I R, T H$ and $A G$ reviewed and revised it critically and approved the final version for submission. LAMB and TER are the guarantors.

Funding This research project was reviewed and has been granted funding by the South-Eastern Norway Regional Health Authority (no. 16/00451-33).

Competing interests None declared.

Patient consent for publication Not required.

Ethics approval The research project has received ethical approval from the Regional Committee for Medical and Health Research Ethics (no 2017/1246) and approval from the Norwegian Data Protection Authority. The project is also approved by the NorPD, Cancer Registry of Norway and the MBRN.

Provenance and peer review Not commissioned; externally peer reviewed.

Open access This is an open access article distributed in accordance with the Creative Commons Attribution Non Commercial (CC BY-NC 4.0) license, which permits others to distribute, remix, adapt, build upon this work non-commercially, and license their derivative works on different terms, provided the original work is properly cited, appropriate credit is given, any changes made indicated, and the use is non-commercial. See: http://creativecommons.org/licenses/by-nc/4.0/.

\section{REFERENCES}

1. The Cancer Registry of Norway. Cancer in Norway 2017. 2018. https://www.kreftregisteret.no/en/General/Publications/Cancer-inNorway/cancer-in-norway-2017/ (cited 26 Nov 2018).

2. Karimkhani C, Green AC, Nijsten T, et al. The global burden of melanoma: results from the Global Burden of Disease Study 2015. Br J Dermatol 2017;177:134-40.

3. Anon. IARC monographs on the evaluation of carcinogenic risks to humans. Solar and ultraviolet radiation. IARC Monogr Eval Carcinog Risks Hum 1992;55:1-316.

4. IARC. IARC Working group on the evaluation of carcinogenic risk to humans radiation. Lyon (FR): International Agency for Research on Cancer, 2012.

5. Arnold M, de Vries E, Whiteman DC, et al. Global burden of cutaneous melanoma attributable to ultraviolet radiation in 2012. Int $J$ Cancer 2018;143:1305-14.

6. Berwick M, Buller DB, Cust A. Melanoma Epidemiology and Prevention. In: Kaufman HL, Mehnert JM, eds. Melanoma. Cham: Springer International Publishing, 2016:17-49.

7. Robsahm TE, Karagas MR, Rees JR, et al. New malignancies after squamous cell carcinoma and melanomas: a population-based study from Norway. BMC Cancer 2014;14:210.

8. Hemminki K, Zhang H, Czene K. Familial and attributable risks in cutaneous melanoma: effects of proband and age. $J$ Invest Dermatol 2003;120:217-23.

9. Stenehjem JS, Veierød MB, Nilsen LT, et al. Anthropometric factors and cutaneous melanoma: Prospective data from the populationbased Janus Cohort. Int J Cancer 2018;142:681-90.

10. De Giorgi V, Gori A, Savarese I, et al. Role of BMI and hormone therapy in melanoma risk: a case-control study. J Cancer Res Clin Oncol 2017;143:1191-7.

11. Rota M, Pasquali E, Bellocco R, et al. Alcohol drinking and cutaneous melanoma risk: a systematic review and dose-risk metaanalysis. Br J Dermatol 2014;170:1021-8.

12. IARC. IARC monographs on the evaluation of carcinogenic risks to humans. Agents classified by the IARC monographs, volumes 123. 2018. https://monographs.iarc.fr/agents-classified-by-the-iarc/ (cited 201820 November).

13. Pottegård $\mathrm{A}$, Friis $\mathrm{S}$, Stürmer $\mathrm{T}$, et al. Considerations for Pharmacoepidemiological Studies of Drug-Cancer Associations. Basic Clin Pharmacol Toxicol 2018;122:451-9.

14. Selvåg E. [Drugs and sun]. Tidsskr Nor Laegeforen 2003;123:1860-1.

15. Rønning M, Berg C, Furu K, et al. The Norwegian Prescription Database 2005-2009: Norwegian Institute of Public Health, 2010.

16. Berg C, Blix HS, Fenne O, et al. The Norwegian Prescription Database 2011-2015 (Legemiddelstatistikk 2016:2): Norwegian Institute of Public Health, 2016. 
17. Gandini S, Palli D, Spadola G, et al. Anti-hypertensive drugs and skin cancer risk: a review of the literature and meta-analysis. Crit Rev Oncol Hematol 2018;122:1-9.

18. Ji Y, Chen S, Xiao X, et al. $\beta$-blockers: a novel class of antitumor agents. Onco Targets Ther 2012;5:391-401.

19. De Giorgi V, Grazzini M, Gandini S, et al. Treatment with $\beta$-blockers and reduced disease progression in patients with thick melanoma. Arch Intern Med 2011;171:779-81.

20. De Giorgi V, Gandini S, Grazzini M, et al. Effect of $\beta$-blockers and other antihypertensive drugs on the risk of melanoma recurrence and death. Mayo Clin Proc 2013;88:1196-203.

21. Lemeshow S, Sorensen HT, Phillips G, et al. -Blockers and Survival among Danish patients with malignant melanoma: a populationbased cohort study. Cancer Epidemiology Biomarkers \& Prevention 2011;20:2273-9.

22. Tang H, Fu S, Zhai S, et al. Use of Antihypertensive Drugs and Risk of Malignant Melanoma: A Meta-analysis of Observational Studies. Drug Saf 2018;41:161-9.

23. Monteiro AF, Rato M, Martins C. Drug-induced photosensitivity: photoallergic and phototoxic reactions. Clin Dermatol 2016;34:571-81.

24. Pedersen SA, Gaist D, Schmidt SAJ, et al. Hydrochlorothiazide use and risk of nonmelanoma skin cancer: A nationwide case-control study from Denmark. J Am Acad Dermatol 2018;78:673-81.

25. Jensen $A \varnothing$, Thomsen HF, Engebjerg MC, et al. Use of photosensitising diuretics and risk of skin cancer: a populationbased case-control study. Br J Cancer 2008;99:1522-8.

26. Nardone B, Majewski S, Kim AS, et al. Melanoma and NonMelanoma Skin Cancer Associated with Angiotensin-ConvertingEnzyme Inhibitors, Angiotensin-Receptor Blockers and Thiazides: A Matched Cohort Study. Drug Saf 2017;40:249-55.

27. Pottegård A, Pedersen SA, Schmidt SAJ, et al. Association of hydrochlorothiazide use and risk of malignant melanoma. JAMA Intern Med 2018;178:1120-2.

28. Koomen ER, Joosse A, Herings RM, et al. Is statin use associated with a reduced incidence, a reduced Breslow thickness or delayed metastasis of melanoma of the skin? Eur J Cancer 2007;43:2580-9.

29. Alvarenga EC, Fonseca MC, Carvalho CC, et al. Angiotensin Converting Enzyme Regulates Cell Proliferation and Migration. PLOS One 2016;11:e0165371.

30. Mc Menamin ÚC, Murray LJ, Cantwell MM, et al. Angiotensinconverting enzyme inhibitors and angiotensin receptor blockers in cancer progression and survival: a systematic review. Cancer Causes Control 2012;23:221-30.

31. de Vries E, Trakatelli M, Kalabalikis D, et al. Known and potential new risk factors for skin cancer in European populations: a multicentre case-control study. Br J Dermatol 2012;167(Suppl. 61):1-13.

32. Steingart $A B$, Cotterchio $M$. Do antidepressants cause, promote, or inhibit cancers? J Clin Epidemiol 1995;48:1407-12.

33. Reddy KK, Lefkove B, Chen LB, et al. The antidepressant sertraline downregulates Akt and has activity against melanoma cells. Pigment Cell Melanoma Res 2008;21:451-6.

34. Lin CJ, Robert F, Sukarieh R, et al. The antidepressant sertraline inhibits translation initiation by curtailing mammalian target of rapamycin signaling. Cancer Res 2010;70:3199-208

35. Liao S, Shang J, Tian X, et al. Up-regulation of melanin synthesis by the antidepressant fluoxetine. Exp Dermatol 2012;21:635-7.

36. Shapovalov Y, Zettel M, Spielman SC, et al. Fluoxetine modulates breast cancer metastasis to the brain in a murine model. BMC Cancer 2014;14:598

37. Grygier B, Arteta B, Kubera M, et al. Inhibitory effect of antidepressants on B16F10 melanoma tumor growth. Pharmacol Rep 2013;65:672-81.

38. Kirkova M, Tzvetanova E, Vircheva S, et al. Antioxidant activity of fluoxetine: studies in mice melanoma model. Cell Biochem Funct 2010;28:497-502.
39. Parker KA, Glaysher S, Hurren J, et al. The effect of tricyclic antidepressants on cutaneous melanoma cell lines and primary cell cultures. Anticancer Drugs 2012;23:65-9.

40. Kubera M, Grygier B, Arteta B, et al. Age-dependent stimulatory effect of desipramine and fluoxetine pretreatment on metastasis formation by B16F10 melanoma in male C57BL/6 mice. Pharmacol Rep 2009:61:1113-26.

41. Halloran PF. Immunosuppressive drugs for kidney transplantation. $N$ Engl J Med Overseas Ed 2004;351:2715-29.

42. Rizvi SMH, Aagnes B, Holdaas H, et al. Long-term change in the risk of skin cancer after organ transplantation: a population-based nationwide cohort study. JAMA Dermatol 2017;153:1270-7.

43. Green AC, Olsen CM. Increased risk of melanoma in organ transplant recipients: systematic review and meta-analysis of cohort studies. Acta Derm Venereol 2015;95:923-7.

44. Nardone B, Hammel JA, Raisch DW, et al. Melanoma associated with tumour necrosis factor- $\alpha$ inhibitors: a Research on Adverse Drug events And Reports (RADAR) project. Br J Dermatol 2014;170:1170-2.

45. Banciu M, Schiffelers RM, Fens MH, et al. Anti-angiogenic effects of liposomal prednisolone phosphate on B16 melanoma in mice. $J$ Control Release 2006;113:1-8.

46. Bhakoo HS, Paolini NS, Milholland RJ, et al. Glucocorticoid receptors and the effect of glucocorticoids on the growth of B16 melanoma. Cancer Res 1981;41:1695-701.

47. O'Donovan P, Perrett CM, Zhang X, et al. Azathioprine and UVA light generate mutagenic oxidative DNA damage. Science 2005;309:1871-4.

48. Robbins HA, Clarke CA, Arron ST, et al. Melanoma Risk and Survival among Organ Transplant Recipients. J Invest Dermatol 2015;135:2657-65.

49. Larsen IK, Småstuen M, Johannesen TB, et al. Data quality at the Cancer Registry of Norway: an overview of comparability, completeness, validity and timeliness. Eur J Cancer 2009;45:1218-31.

50. Furu K, Wettermark B, Andersen M, et al. The Nordic countries as a cohort for pharmacoepidemiological research. Basic Clin Pharmacol Toxicol 2010;106:86-94.

51. WHO Collaborating Centre for Drug Statistics Methodology. Guidelines for ATC classification and DDD assignment 2015: Norwegian Institute of Public Health, 2016.

52. Stenehjem JS, Grimsrud TK, Rees JR, et al. A protocol for prospective studies of 25-hydroxyvitamin D, leptin and body mass index in relation to cutaneous melanoma incidence and survival. BMJ Open 2017;7:e014829.

53. Yoshida K, Solomon DH, Kim SC. Active-comparator design and new-user design in observational studies. Nat Rev Rheumato 2015;11:437-41.

54. Pottegård $\mathrm{A}$, Hallas J. New use of prescription drugs prior to a cancer diagnosis. Pharmacoepidemiol Drug Saf 2017;26:223-7.

55. Green AC, Williams GM, Logan V, et al. Reduced melanoma after regular sunscreen use: randomized trial follow-up. J Clin Oncol 2011;29:257-63.

56. Gershenwald JE, Scolyer RA, Hess KR, et al. Melanoma staging: Evidence-based changes in the American Joint Committee on Cancer eighth edition cancer staging manual. CA Cancer J Clin 2017;67:472-92

57. Core Team R. R: A language and environment for statistical computing. Vienna, Austria: R Foundation for Statistical Computing, 2017.

58. Pottegård A, Christensen R, Houji A, et al. Primary non-adherence in general practice: a Danish register study. Eur J Clin Pharmacol 2014;70:757-63

59. Yachida S, Jones S, Bozic I, et al. Distant metastasis occurs late during the genetic evolution of pancreatic cancer. Nature 2010;467:1114-7. 